\title{
Computer Simulation of Displacement Cascades in Nanocrystalline Ni
}

\author{
M. Samaras, ${ }^{1}$ P. M. Derlet, ${ }^{1}$ H. Van Swygenhoven, ${ }^{1}$ and M. Victoria ${ }^{2}$ \\ ${ }^{1}$ Paul Scherrer Institute, CH-5232 Villigen, Switzerland \\ ${ }^{2}$ CRPP-Fusion Technology Materials, EPFL, CH-5232 Villigen-PSI, Switzerland \\ (Received 2 October 2001; published 7 March 2002)
}

\begin{abstract}
Large-scale molecular dynamics of cascade production of the primary damage state are performed in fcc nanocrystalline $\mathrm{Ni}$ of average grain diameters of 5 and $12 \mathrm{~nm}$. Primary knock-on atom kinetic energies of $5-30 \mathrm{keV}$ are simulated. During the thermal spike phase, significant atomic motion towards the surrounding grain boundary structure is observed, characterized by many replacement-collision sequences. Upon resolidification, the excess volume condenses to form vacancy dominated defects with a complex partial dislocation network forming at higher energies.
\end{abstract}

DOI: $10.1103 /$ PhysRevLett.88.125505

The reduction of grain size down to the nanometer regime has opened new and fascinating avenues for research in several aspects of material science [1]. At the lower end of the grain size range obtainable nowadays, up to $30 \%$ of the atoms belong to or are affected by the presence of interfaces. Grain boundaries (GBs) play a predominant role in the deformation of such materials, although the details of the precise deformation mechanism at the atomic level is still under debate [2,3]. Nanocrystalline (nc) materials are also believed to exhibit quite different responses under irradiation environments compared to their coarser-grained counterparts. Measurements in ion irradiated samples $[4,5]$ at $300 \mathrm{~K}$ have shown that the defect production is significantly smaller than its polycrystalline counterpart with the nc rapidly saturating in terms of dose.

The proton and neutron irradiation of materials can result in important microstructural changes with correspondingly significant changes in the mechanical properties [6]. Such changes in the atomic scale structure begin with the so-called primary damage state and its subsequent evolution at a variety of different time and length scales. The primary damage state, however, arises from processes highly localized in both time and space, and is therefore amenable to the molecular dynamics (MD) technique. The use of MD has led to significant advances in the understanding of the early dynamics of this phase [7]. MD simulations have predominantly been performed on perfect fcc/bcc/hcp geometries of metals and alloys, allowing for atomic scale characterization of vacancy and interstitial clusters [8]. The initial microstructure of the target material will play an important role in the resulting defect/damage character as a function of dose, with grain boundaries [9], preexisting defects [10], and surfaces [11,12] acting as sinks for mobile defects. Interstitial loss to nearby GBs has been thought of as a possible cause of enhanced void swelling in this region $[13,14]$. In polycrystalline material, where grain sizes are large compared to the characteristic diffusion length of mobile defects, comparison with perfect lattice geometries is justified. However, in the nanoscale regime, where grain sizes are comparable or even smaller than the maximum cascade volumes, such an approach is no longer warranted.
PACS numbers: $61.80 . \mathrm{Hg}, 02.70 . \mathrm{Ns}, 81.07 . \mathrm{Bc}$

In this Letter, we report on large-scale MD simulations of displacement cascades in nc-Ni. We present the results of 5, 20, and $30 \mathrm{keV}$ primary knock-on atom (PKA) $\mathrm{Ni}$ atoms in samples with mean grain sizes of 5 (one cascade) and $12 \mathrm{~nm}$ (three cascades). It is shown that the GB acts as a preferential sink for energetic atoms, changing the character of the defects created by the cascade when compared to single crystals. The nc-Ni samples used in the present work have been employed extensively in previous studies of grain boundary structure and their deformation properties [15-18]. They contain a fully three-dimensional grain boundary network based on the Voronoi construction [19], which is believed to contain the essential geometry of nc materials. Each configuration contains approximately $1.2 \times 10^{6}$ atoms, corresponding to 125 grains for the $5 \mathrm{~nm}$ grain size sample and 15 grains for the $12 \mathrm{~nm}$ sample. The details of their preparation can be found in [17]. For this previous work we used the second moment tight binding potentials of Cleri and Rosato [20]. Here the short-range form of these potentials was modified to reproduce the high-energy empirical potential of Ziegler et al. [21].

The PKA simulations were performed at room temperature in a 3D-MD box, using periodic boundary conditions at constant volume. Heat was extracted via velocity rescaling of atoms within an outer cubic shell at the periodic boundary. The PKA was introduced at approximately the center of the MD box, located at an edge of a grain with its velocity vector pointing inward to the grain. For comparison, each PKA energy and direction was repeated for a perfect fcc Ni lattice of comparable size. Similar work has been done on single crystal Ni by Almazouzi et al. [22]. As with the previous work, grain boundary and cascade visualization is aided by identifying the local crystalline order of each atom using a short-range topological analysis [23], and representing fcc atoms as grey, 12-coordinated non-fcc and non-12-coordinated atoms as green and blue, and first nearest neighbor hcp coordinated atoms as red circles.

Figure 1a displays a cross section of a grain containing a $5 \mathrm{keV}$ cascade in the $12-\mathrm{nm} \mathrm{nc}$ sample, at $14.15 \mathrm{ps}$, after the introduction of the PKA. The surrounding grain 


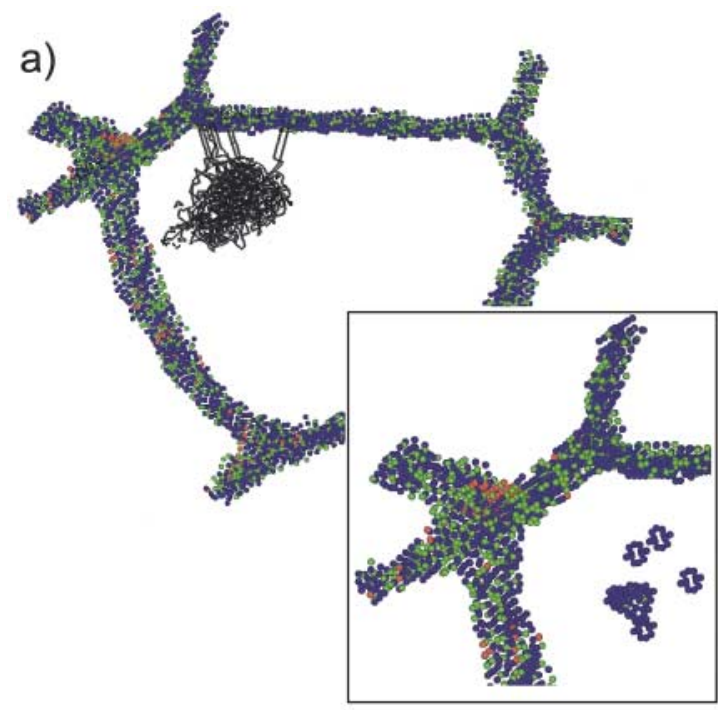

b)

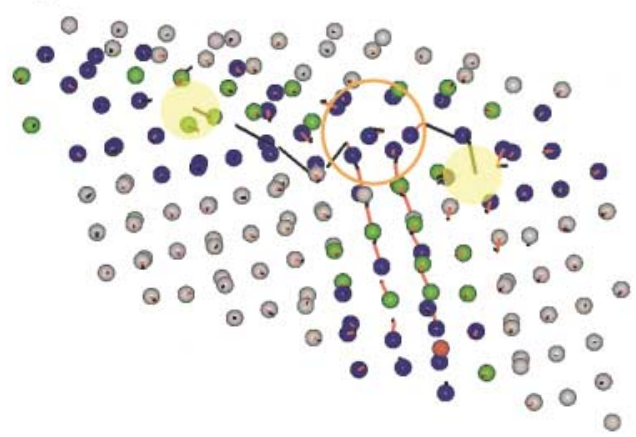

FIG. 1 (color). (a) Selected area of the 12-nm nc grain size sample showing the grain boundary (GB) atoms and the displacement vectors $(>1.5 \AA)$ between the atoms due to a $5 \mathrm{keV}$ PKA. The inset shows a magnified view of the defect region after cooling down. (b) An example of the GB acting as an interstitial sink, by the annihilation of (RCS) interstitials with free volume in the GB (marked in yellow).

boundary structure is pictured by green, blue, and red atoms, while the thermal spike region is visualized by showing the displacement vectors between atomic positions before the introduction of the PKA and after at 14.15 ps. We note that the maximum cascade volume was found to be approximately the size of that in the perfect crystal, both occurring within $\sim 400$ fs of the PKA introduction. Inspection of the figure reveals a "funnelling" of the cascade towards the grain boundary region. This can be seen by the large number of replacement collision sequences (RCSs) that are directed towards the grain boundary region. These mostly occurred between 0.5 and 4 ps after the introduction of the PKA, with some occurring up until 9 ps (see Fig. 1b). This is a common observation for all cascades performed in the nc sample at different PKA energies. Such collective and directed RCS activity was not observed in the perfect fcc Ni simulations, suggesting that the presence of the GB provides optimal conditions for this process to occur. The inset of Fig. 1a displays a magnified view of the defect structure at 14.15 ps. These non-fcc atoms are mainly of reduced coordination, indicating a number of single vacancy defects and one extended vacancy cluster. Thus, upon condensation of the thermal spike, a defect structure containing no interstitials resulted. For the perfect fcc, both vacancy and interstitial-like cluster defects could be observed. Thus the GB acts as a sink for interstitials, where direct transport to the GB is predominantly via the RCS mechanism.

Figure 1b displays in more detail two RCSs collectively arriving at the GB. Here the red lines represent the atomic displacement vectors from 7.35 ps (after the PKA is introduced) to $8.95 \mathrm{ps}$, and the black, from 8.95 ps to $14.15 \mathrm{ps}$. The atomic positions are those of the configuration at 7.35 ps. The blue and green atoms within the lower grain arise from the disorder of the cooling thermal spike region, and those separating the two fcc regions (grains) indicate the GB region. Upon the arrival of two interstitials at the GB, from the RCSs, the continuation of the RCS within the GB structure can be observed. This is evident through observation of the circled atoms, which, due to the presence of the interstitials, relax within the GB, after which the RCS process continues within the GB until annihilation with neighboring free volume (marked in yellow). Thus, some structural relaxation exists within the grain boundary to accommodate the atomic transport; however, in general no significant grain boundary migration was observed.

Figure 2 represents an area in the $12 \mathrm{~nm}$ nc sample in which a $20 \mathrm{keV}$ PKA has been introduced. In this case subcascade formation was seen, where one of the secondary cascades nucleated precisely at a triple junction (TJ) region. The primary cascade can be seen inside a (lower) neighboring grain. In Fig. 2a, the surrounding grain boundary structure is pictured by the non-fcc atoms, while the thermal spike region is visualized by showing the displacement vectors $(>1.5 \AA)$ between atomic positions before the introduction of the PKA and after at 55.63 ps. We note that the maximum volume of the primary and subcascade are approximately equal (occurring at $\sim 800 \mathrm{fs}$ ), where the subcascade formation occurred in the first $500 \mathrm{fs}$ after the introduction of the PKA via the emission of an energetic atom from the primary cascade in the first $100 \mathrm{fs}$ of cascade evolution. The many RCSs occur primarily in the sub-2 ps regime. Figure $2 \mathrm{~b}$ shows the configuration after cooling (at $55.63 \mathrm{ps)}$ where the colored circles represent vacancy defects within the grain. Here the subcascade sitting on the TJ does not create defects after cooling, whereas in the primary cascade region, vacancies and vacancy clusters are left behind, very similar to the cascade described in Fig. 1. For the analogous perfect fcc PKA simulation after cooling down, both vacancy and interstitial-like cluster defects, as well as sessile interstitial dislocation loops, could be seen.

Figure 3 represents a grain in the $12 \mathrm{~nm}$ sample where cascade formation occurred via a $30 \mathrm{keV}$ PKA. For such a PKA energy, the maximum volume of the cascade is typically about the size of the grain. In Fig. 3a the 

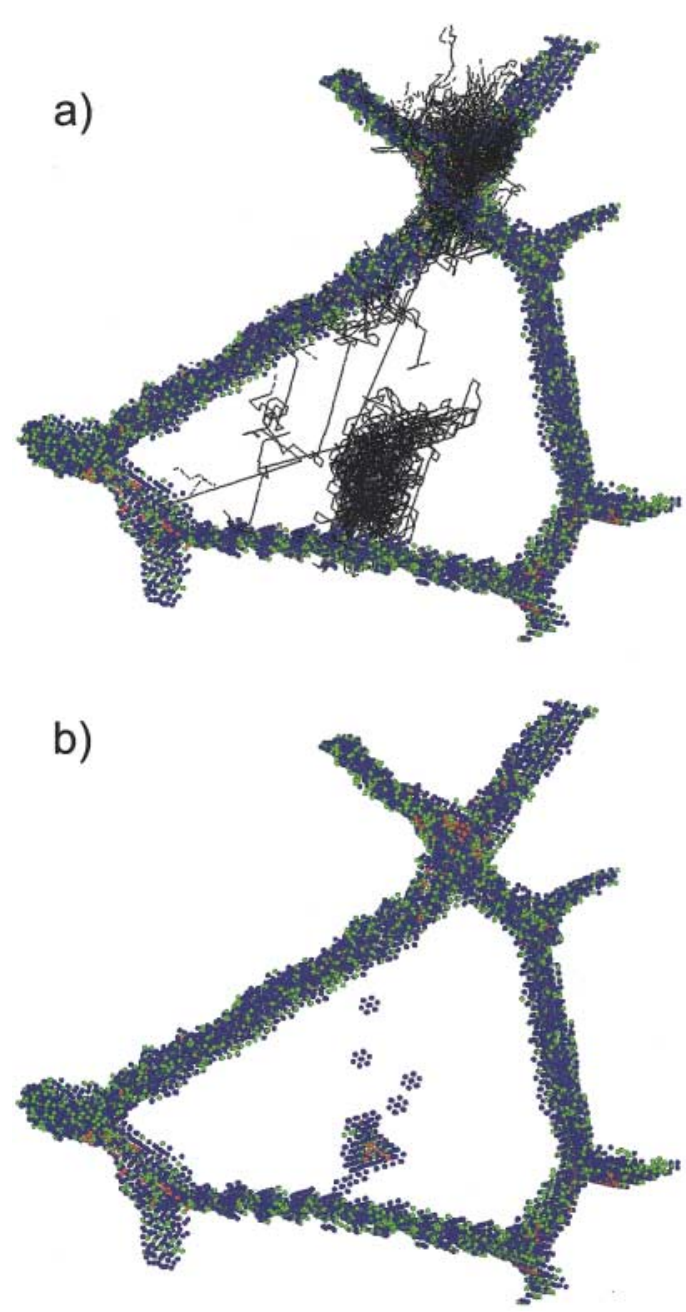

FIG. 2 (color). (a) Displays the displacement vectors $(>1.5 \AA)$ between the atoms due to a $20 \mathrm{keV}$ PKA in a $12-\mathrm{nm}$ nc grain size sample. (b) The same region after the cascade has cooled in which the damage at the triple junction has been completely removed, whereas the lower subcascade (which exists entirely in a grain) condenses to a vacancy defect concentration.

displacement vectors are shown between the initial positions and those at $47.92 \mathrm{ps}$ after the PKA, where the cascade has cooled. Again there is evidence of funnelling via the RCS mechanism, facilitating atomic transport towards the nearby GB structure. Figure $3 b$ represents the view after the thermal spike region has cooled. Here the red atoms show that after cooling, many pairs of adjacent (111) hcp planes exist within the grain, indicating the presence of stacking faults and thus partial dislocation activity. Dislocation locks are present, suggesting that a number of slip planes have been active. Upon closer inspection we found that the stacking faults occurred within the maximum cascade volume, and also at the edge in the region where large stress gradients are present due to the pressure front of the thermal spike region. Atomic transport (via RCS) towards the GB primarily in the first 2 ps induces a significant amount of free volume within the grain. Upon resolidification, the partial dislocation network accommodates much of this $(>95 \%)$, with the remaining volume
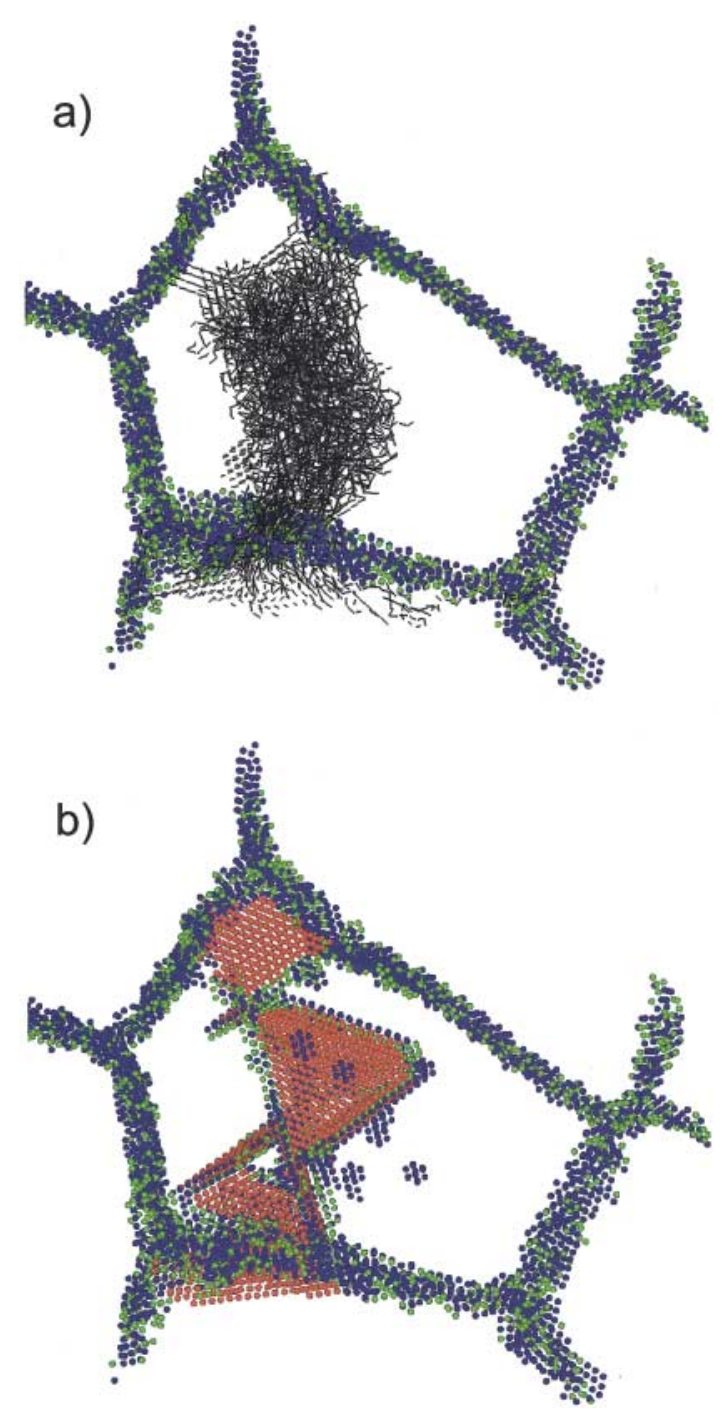

FIG. 3 (color). (a) Selected area of the $12 \mathrm{~nm} \mathrm{nc}$ grain size sample showing the non-fcc atoms and the displacement vectors $(>1.5 \AA)$ between the atoms due to a $30 \mathrm{keV}$ PKA. (b) The non-fcc atomic positions after the cascade has cooled, indicating a partial dislocation network emerges.

existing as a small number of isolated vacancy defects. In one case slip was observed via the nucleation of a partial at a neighboring GB, which subsequently propagated into the grain. Figure 4 shows a similar cascade after cooling down in the $5 \mathrm{~nm}$ grain size sample, where the cascade volume typically covers up to three grains. The approximate boundary of the maximum cascade volume is also indicated in yellow. As with the $12 \mathrm{~nm}$ sample, the region of dislocation activity after cooling down correlates well with the maximum volume of the cascade; indeed, hcp planes begin forming at approximately 3 ps of cascade evolution, with the final stacking fault structure being formed by approximately $13.5 \mathrm{ps}$. We note that partial dislocation activity has been observed as a deformation mechanism in MD simulations of nc-fcc metals [15-18,24]. For the fcc case, no such extended partial dislocation structures were observed, the structure upon cooling consisting of vacancy and interstitial-like defects. 


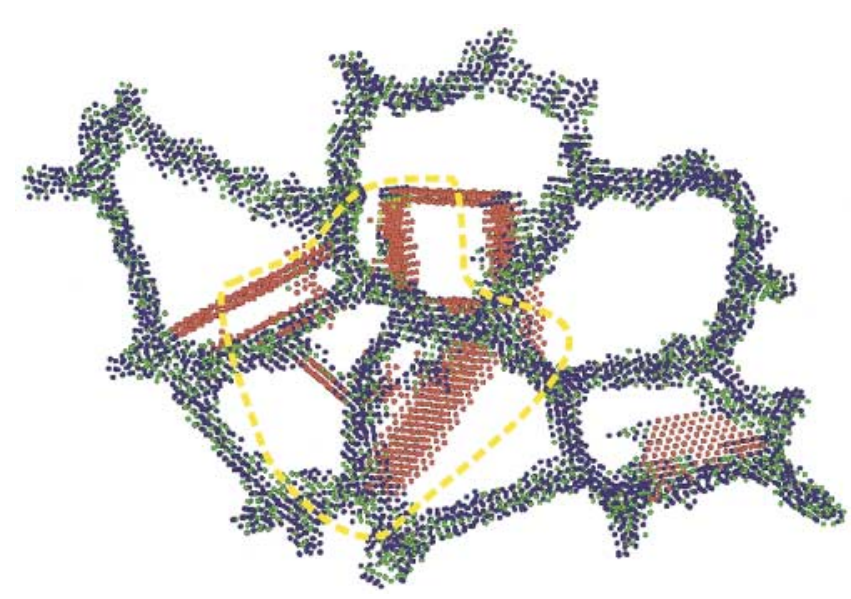

FIG. 4 (color). A selected area of the 5-nm grain size sample in which a $30 \mathrm{keV}$ PKA was introduced, showing that upon resolidification a complex partial dislocation network exists over a number of grains.

We have demonstrated that at the nanoscale, the GB plays an important role in the resulting damage and defect structure of the primary damage state due to proton and neutron irradiation. In the presence of a nearby GB, the liquidlike core region and subsequent thermal spike region tend to funnel towards the GB, with mass transport occurring via the RCS mechanism. This leaves behind in the grain a defect structure that is mainly based on vacancies. The number of vacancies in the nc is larger than in the single crystal as the interstitials are lost to the GB and cannot recombine with vacancies. Such activity has also been observed in low-energy PKA cascade formation near a $\Sigma=5 \mathrm{~GB}$ in $\mathrm{Ag}$ [9], where significant directed atom transport occurred via interstitial migration due to the strain field associated with the GB. In that work the mean-square displacement of interstitials was seen to be a factor of 2 larger than that of a single crystal. For the higher PKA energies, we found that in the nc, there exists a resulting dislocation network, which accommodates most of the vacancy population created by the mass transport to the surrounding GB structure. Analogous dislocation activity has also been observed in MD simulations of cascade formation near an $\mathrm{Au}(100)$ surface $[7,11,12]$. In that case, however, a "necking" was produced by directed melting of the cascade towards the surface involving the production of a large number of adatoms. Upon resolidification, a complex dislocation network forms within $10 \mathrm{~nm}$ of the surface. In general, such complex and extended dislocation networks are not observed experimentally in bulk single crystal fcc systems [25].

In conclusion, the present work demonstrates that the GB structure at the nanoscale regime strongly affects the primary damage state, with the GB acting as an interstitial sink and thus allowing for, after resolidification, a vacancy dominated defect structure which in some cases can be completely removed by the surrounding GB/TJ regions. This is quite different from the single crystal result where the primary damage state must arise from a vacancy/ interstitial defect distribution. For higher PKA energies, a complex partial dislocation network arises that in part accommodates the reduced atom density within the grain due to the atomic transport towards the GB.

M. S. acknowledges the financial support of the BBW (Grant No. 98.0098), and M. V. acknowledges the financial support of the Swiss-FN (Grant No. 2000-061837.00). The authors thank M.J. Caturla and B.D. Wirth at Lawrence Livermore National Laboratory for their valuable discussion and assistance which took part within a collaboration agreement between the two research groups.

[1] H. Gleiter, Acta Mater. 48, 1 (2000).

[2] C. C. Koch et al., MRS Bull. 24, 54 (1999).

[3] J. R. Weertman et al., MRS Bull. 24, 44 (1999).

[4] M. Rose, A. G. Balogh, and H. Hahn, Nucl. Instrum. Methods Phys. Res., Sect. B 127/128, 119 (1997).

[5] Y. Chimi et al., J. Nucl. Mater. 297, 355 (2001).

[6] M. Victoria et al., J. Nucl. Mater. 276, 114 (2000).

[7] R. S. Averback and T. Diaz de la Rubia, in Solid State Physics, edited by F. Spaepen and H. Ehrenreich (Academic Press, New York, 1998), Vol. 51, pp. 281-402.

[8] D. J. Bacon, F. Gao, and Yu. N. Osetsky, Nucl. Mater. 276, 1 (2000).

[9] K. Sugio, Y. Shimomura, and T. Diaz de la Rubia, J. Phys. Soc. Jpn. 67, 882 (1998).

[10] C. A. English, W. J. Phythian, and A. J. E. Foreman, J. Nucl. Mater. 174, 135 (1990).

[11] M. Ghaly and R. S. Averback, Phys. Rev. Lett. 72, 364 (1994).

[12] R.S. Averback and M. Ghaly, J. Appl. Phys. 76, 3908 (1994).

[13] A. J.E. Foreman, B. N. Singh, and A. Horsewell, Mater. Sci. Forum 15-18, 895 (1987).

[14] H. Trinkhaus, B. N. Singh, and A. J. E. Foreman, J. Nucl. Mater. 206, 200 (1993).

[15] H. Van Swygenhoven, D. Farkas, and A. Caro, Phys. Rev. B 62, 831 (2000).

[16] H. Van Swygenhoven and A. Caro, Appl. Phys. Lett. 71, 1652 (1997).

[17] H. Van Swygenhoven, M. Spaczer, and A. Caro, Acta Mater. 47, 3117 (1999).

[18] H. Van Swygenhoven and P. M. Derlet, Phys. Rev. B 64, 224105 (2001).

[19] G. Z. Voronoi and J. Reine, Angew. Math. 134, 199 (1908).

[20] F. Cleri and V. Rosato, Phys. Rev. B 48, 22 (1993).

[21] J. Ziegler, J. P. Biersack, and U. Littmark, in The Stopping and Range of Ions in Solids (Pergamon, New York, 1987). See also S. Pronnecke et al., J. Mater. Res. 6, 483 (1991).

[22] A. Almazouzi et al., Mater. Res. Soc. Symp. Proc. 540, 685 (1999).

[23] D. J. Honeycutt and H. C. Andersen, J. Phys. Chem. 91, 4950 (1987).

[24] J. Schiøtz, F. D. Di Tolla, and K. W. Jacobsen, Nature (London) 391, 561 (1998).

[25] B. N. Singh and S. J. Zinkle, J. Nucl. Mater. 206, 212 (1993). 